ated the potential for side effects by testing the monoclonal antibody on three healthy primates. The primate studies with the new anti-DR reagent are highly reassuring that the expression of DR on a variety of non-malignant cell types, though limited, will not be clinically limiting.

Two monoclonal antibodies with specificity for some haplotypes of human DR $\beta$-chains have been used in humans and one of these, a humanized antibody called apolizumab (Remitogen) is in phase 2 clinical trials ${ }^{11}$. It is not clear whether either of the antibodies tested so far can mediate signal transduction. However, the reagent reported by Nagy and colleagues certainly does and is also more broadly reactive with the range of human DR polymorphisms. The promise of the new approach is that cell death is a direct consequence of antibody binding to the target on tumor cells, and it does not require additional mechanisms that may be compromised in the cancer patient. Like chemotherapeutic agents and radiation therapy, the new antibody against HLA-DR is directly cytotoxic.

Despite promising data such as these it is dangerous to make predictions about clinical success in cancer treatment. The tumor-cell lines killed by the new antibody all have the phenotype of activated $B$ cells and have a growth fraction of $100 \%$, which means all the cells were proliferating. The one cell line that failed to be killed could be rendered susceptible to killing by activation signals. By contrast, human lymphomas have a smaller growth fraction in vivo ( $8 \%$ or fewer of the tumor cells are actively proliferating). In humans, molecular characterization is defining distinct subgroups ${ }^{12}$ that may or may not be susceptible to DR activation in vivo. However, there is every reason to think that antibodies capable of delivering signals through cell-surface receptors will be more effective than antibodies that either block function or recognize a structure that serves no functional role in the activation and growth of the tumor cell. The next step is to take the new weapon into the clinic. If it is as successful as expected, the path to the generation of other effective new treatments will be well illuminated by this elegant work.

1. Nagy, Z.A. et al. Fully human, HLA-DR-specific monoclonal antibodies efficient induce programmed death of malignant lymphoid cells. Nature Med. 8, 801-807 (2002).

2. Nadler, L.M. et al. Serotherapy of a patient with a monoclonal antibody directed against a human lymphoma-associated antigen. Cancer Res. 40, 3147-3154 (1980).

3. Scott, D.W. et al. Lymphoma models for B-cell activation and tolerance. II. Growth inhibition by anti-mu of WEHI-231 and the selection and properties of resistant mutants. Cell Immunol. 93,
124-131 (1985)

4. Vuist, W.M. et al. Lymphoma regression induced by monoclonal anti-idiotypic antibodies correlates with their ability to induce Ig signal transduction and is not prevented by tumor expression of high levels of bcl-2 protein. Blood 83, 899-906 (1994).

5. Ashwell, J.D., Longo, D.L. \& Bridges, S.H. T-cell tumor elimination as a result of T-cell-receptormediated activation. Science 237, 61-64 (1987).

6. Funakoshi, S. et al. Inhibition of human B-cell lymphoma growth by CD40 stimulation. Blood 83, 2787-2794 (1994).

7. Tian, Z.G. et al. In vivo antitumor effects of unconjugated CD30 monoclonal antibodies on human anaplastic large-cell lymphoma xenografts. Cancer Res. 55, 5335-5341 (1995).

8. Alas, S. \& Bonavida, B. Rituximab inactivates signal transducer and activation of transcription 3 (STAT3) activity in B-non-Hodgkin's lymphoma through inhibition of the interleukin 10 autocrine/paracrine loop and results in down-regulation of $\mathrm{Bcl}-2$ and sensitization to cytotoxic drugs. Cancer Res 61, 5137-5144 (2001).

9. Bridges, S.H., Kruisbeek, A.M. \& Longo, D.L. Selective in vivo antitumor effects of monoclonal anti-I-A antibody on B-cell lymphoma. J. Immunol. 139, 4242-4249 (1987).

10. Knappik, A. et al. Fully synthetic human combinatorial antibody libraries (HuCAL) based on modular consensus frameworks and CDRs randomized with trinucleotides. J. Mol. Biol. 296, 57-86 (2000).

11. Brown, K.S., Levitt, D.J., Shannon, M. \& Link, B.K. Phase II trial of Remitogen (humanized 1D10) monoclonal antibody targeting class II in patients with relapsed low-grade or follicular lymphoma. Clin. Lymphoma 2, 188-190 (2001).

12. Rosenwald, A. et al. The use of molecular profiling to predict survival after chemotherapy for diffuse large-B-cell lymphoma. N. Engl. J. Med. 346, 1937-1947 (2002).

National Institute on Aging

Baltimore, Maryland, USA

Email:longod@grc.nia.nih.gov

\title{
Host genetics and HIV—removing the mask
}

\author{
A newly discovered interplay between two human genes controlling the immune response to HIV has implications \\ for antiretroviral drug therapy and the development of preventive vaccines.
}

Studies of the impact of host genet$\checkmark$ ics on the susceptibility to HIV infection and rate of disease progression have revealed associations with a number of individual genes. These include those genes encoding proteins that control viral entry (CCR5, CCR2, RANTES and SDF1), immune regulation (interleukin-10, tumor necrosis factor- $\alpha$ and MBL) and adaptive immune recognition by $\mathrm{T}$ cells (human leukocyte antigen or HLA) $)^{1,2}$. Associations have not yet been described with genes that control natural killer (NK) cells of the innate immune response.

In the August issue of Nature Genetics, Martin et al. describe a complex interplay between genes that control NK cell activity and HLA genes

\section{NELSON L. MichaEL}

that control immune recognition ${ }^{3}$. They find that the influence of the former genes masks that of the latter. This masking, or 'epistasis', represents the first such interplay between host genes to be associated with HIV disease progression. Identification of such an association advances the field to the next level in defining host genetic influences on HIV pathogenesis. This research also implicates a key interaction between the innate and adaptive arms of the cellular immune response in controlling HIV replication.

Viral pathogens are targeted by diverse immune-effector mechanisms.
Cytotoxic T-lymphocytes (CTLs) represent the adaptive immune response. They control the expansion of antigen-specific cellular clones, which recognize viral antigens displayed on infected target cells by $H L A$ class I gene products. Previous exposure to antigen provides for a more rapid immune response upon re-exposure. NK cells are part of the innate immune response, as previous antigen exposure does not modulate NK pathogen recognition. NK cells express cell-surface killer immunoglobulin-like receptors (KIRs) that recognize HLA class I molecules on infected target cells. KIRs can both stimulate and inhibit NK activity ${ }^{4}$. Thus, a mechanistically cogent but complex interplay occurs between $H L A$ and KIR genes in the host im- 


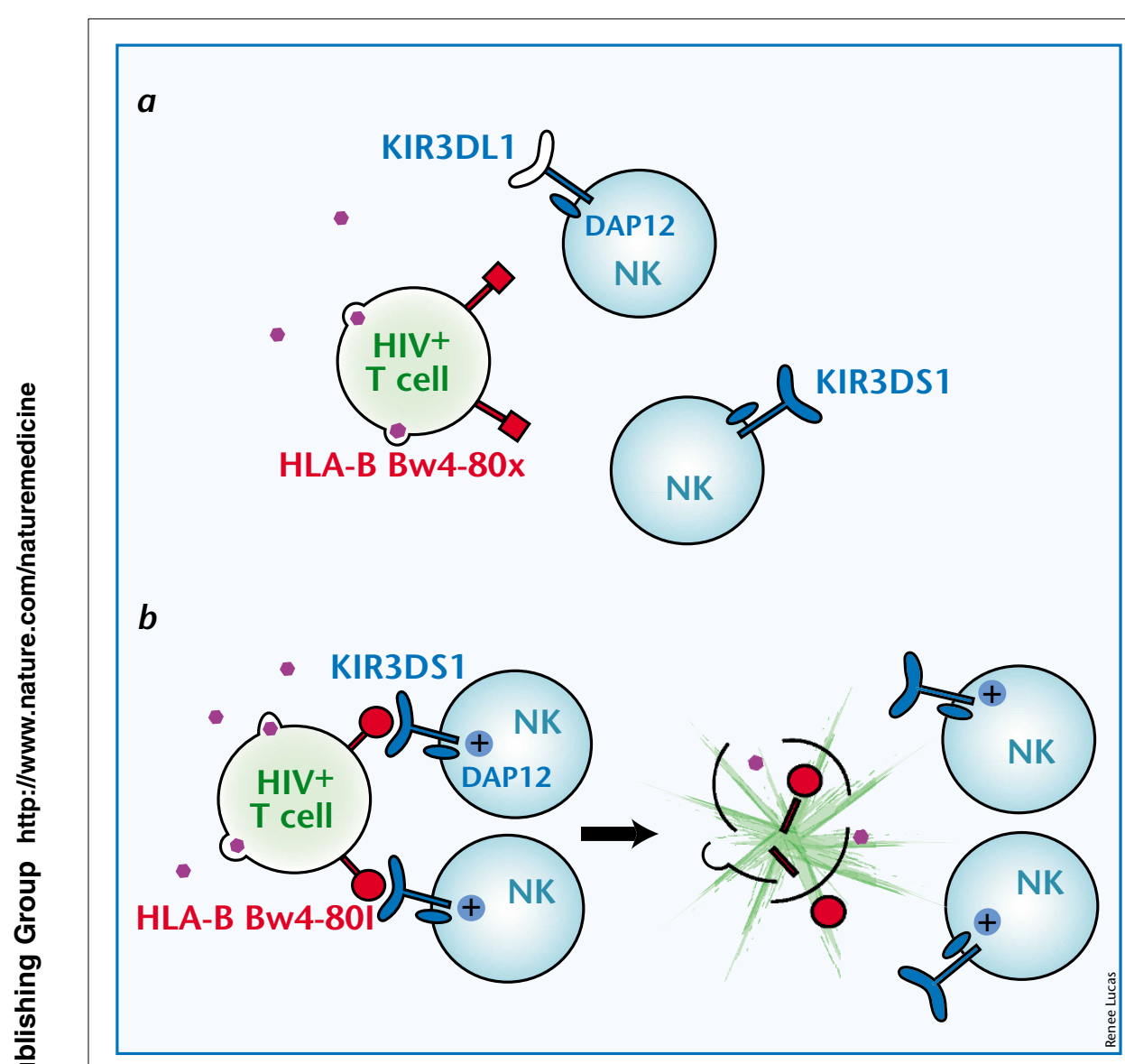

Fig. 1 A proposed mechanism of interaction between $H L A-B \quad B W 4-80 I$ and KIR3DS1 genotypes. a, HIV-infected T cells expressing non-HLA-B BW4-80I molecules (HLA-B 80X) on the cell surface attract fewer NK cells expressing either KIR3DL1 or KIR3DS1, as either is a ligand for the HLA protein. DAP1 2 is not activated and minmal NK-mediated cytolysis occurs. $\boldsymbol{b}$, HIV-infected T cells expressing $H L A-B B W 4-80 /$ molecules are preferentially engaged by KIR3DS1-expressing NK cells which are activated through transmembrane DAP12. The result is killing of the HIV-infected T cell.

mune response to HIV.

Studies of associations between host genes and HIV have focused on genetic suspects involved in the HIV lifecycle and host immune response. The challenge of these studies is to assess the typically small impact of individual genetic polymorphisms. This requires the analysis of large cohorts of individuals both infected with HIV and those at risk of acquiring it. Subjects must be followed for infection and disease outcomes, respectively, for many years.

Initial host gene identification efforts were largely limited to associations of HIV pathogenesis with HLA class I and II genes located on chromosome 9 (ref. 1). The discovery of chemokine receptors as HIV-1 entry coreceptors $^{5}$ generated numerous reports that associated genetic polymorphisms in single chemokine and chemokine receptor genes with HIV pathogenesis. The two most powerful gression after normalizing for the influence of previously associated genotypes such as CCR5 332 .

Martin et al. drew four conclusions. First, they noted that $H L A-B B w 4$ exerted a weak protective effect on AIDS progression when $K I R$ genes were not accounted for. This effect was strictly limited to class $\mathrm{B}$ genotypes with isoleucine at position 80 (HLA-B Bw4$80 I$ ). Second, the KIR3DS1 allele was moderately associated with an increased rate of AIDS progression when HLA-B Bw4-80I was not accounted for. Third, individuals with both KIR3DS1 and $H L A-B B w 4-80 I$ genotypes show a marked delay in disease progression. This delay was striking as these genotypes, when analyzed independently, had divergent associations with disease progression: inclusion of both KIR3DS1 and HLA-B Bw4-80I in a covariate model eliminated the association of $H L A-B$ Bw4-80I with delayed disease, but enhanced the association of KIR3DS1 with rapid disease progression. Thus, the HLA-B Bw4-80I association was masked by KIR3DS1, making the former epistatic to the latter. Fourth, the combined effect of KIR3DS1 and HLA-B Bw4-80I occurred in both Caucasian and AfricanAmerican individuals representing diverse risk exposures and cohorts. The most striking effect of these combined genotypes on disease progression outcomes was on $\mathrm{CD}^{+}{ }^{+} \mathrm{T}$-cell levels in early-stage HIV disease.

These results are novel as they represent the first association of an epistatic interaction between two human genes that modulate the progression of HIV disease. The results are important as they reinforce the critical interface between the adaptive and innate immune response to HIV. However, the precise mechanism that explains the physiological effect of the $H L A$-KIR epistatic interaction remains speculative (Fig. 1). The KIR3DL1 allele encodes cell-surface NK receptors with long cytoplasmic tails that inhibit NK activity ${ }^{9}$. In contrast, the KIR3DS1 allele encodes a NK receptor with the hallmarks of an NK-cell activator, which possibly acts via the transmembrane adaptor DAP12 (ref. 10). A plausible explanation for the current findings is that HLA-B Bw480I molecules are ligands for the activating KIR3DS1 NK receptor. Thus, HIV-positive individuals with both the HLA-B Bw4-80I and KIR3DS1 genotypes 
would express an HLA-B molecule on their infected target cells that could bind to a KIR receptor capable of increasing NK cell-mediated killing of these cells.

What are the clinical implications of this type of research? A comprehensive map linking host genes-both individually and in concert-to HIV pathogenesis would help predict susceptibility for HIV infection and would provide prognostic information for disease progression. A firmer understanding of the host-pathogen interaction could also guide the optimum use of therapeutics. Drug toxicity and resistance increasingly complicate the management of HIV-infected patients, resulting in a larger fraction of patients with deferred treatment. More rational stratification of treatment intensity could be based on a refined knowledge of which individuals are genetically more susceptible to early immune deterioration and disease. Conversely, it might be possible to optimize treatment interruptions and viral-reservoir depletion for patients with greater natural resistance to disease progression. Increased knowledge of host susceptibility to HIV infection could refine the approach to preventive vaccine development by adjusting vaccine effectiveness for innate predisposition for infection. Lastly, better definition of pre-exposure risk for HIV infection might be helpful in the approach to post-exposure treatment of such individuals.

These optimistic implications of a more refined understanding of host genetics and HIV pathogenesis will be challenged by the difficulty in sorting out small influences of any given gene, and the enormous complications of determining sophisticated interactions between genes. Moreover, we must address the ethical considerations of performing large-scale genetic research that predicts disease outcome and therapeutic response with increasing accuracy. The genetic privacy of HIVinfected patients must be respected to avoid employment or insurance discrimination. Nevertheless, the mask has been removed on the first complex interaction between two host genes and HIV pathogenesis-with the promise of more to come.

1. Martin, M.P. \& Carrington, M. The role of human genetics in HIV-1 infection. in Chemokine Receptors and AIDS. (ed. O'Brien, T.R.) 133-162 (Marcel Dekker, New York, 2002).
2. Michael, N.L. Host genetic influences on HIV-1 pathogenesis. Curr. Opin. Immunol. 11, 466-474 (1999).

3. Martin, M.P. et al. Epistatic interaction between KIR3DS1 and HLA-B delays the progression to AIDS. Nature Genet. 31, 429-434 (2002).

4. Long, E.O. \& Rajagopalan, S. HLA class I recognition by killer cell Ig-like receptors. Semin. Immunol. 12, 101-108 (2000).

5. Feng, Y., Broder, C., Kennedy, P.E. \& Berger, E.A. HIV-1 entry cofactor: functional CDNA cloning of a seven-transmembrane $G$ proteincoupled receptor. Science 272, 872-877 (1996).

6. Liu, R. et al. Homozygous defect in HIV-1 coreceptor accounts for resistance of some multiplyexposed individuals to HIV-1 infection. Cell 86, 367-377 (1996).

7. Gao, X. et al. Effect of a single amino acid change in MHC class I molecules on the rate of progression to AIDS. N. Engl. J. Med. 344, p1668-1675 (2001).

8. Carrington, M., et al. HLA and HIV-1: Heterozygote advantage and $\mathrm{B}^{*} 35-\mathrm{CW}^{*} 04$ disadvantage. Science 283, 1748-1752 (1999).

9. Long, E.O. Regulation of immune responses through inhibitory receptors. Annu. Rev. Immunol. 17, 875-904 (1999).

10. Lanier, L.L. et al. Immunoreceptor DAP12 bearing a tyrosine-based activation motif is involved in activating NK cells. Nature 391, 703-707 (1998).

Department of Molecular Diagnostics and

Pathogenesis

Division of Retrovirology

Walter Reed Army Institute of Research

Rockville, Maryland, USA

Email:nmichael@hivresearch.org

\title{
Erythropoietin strikes a new cord
}

\begin{abstract}
There are currently no effective means to treat acute spinal cord injury, except for glucocorticoids, which produce moderate benefits at best. A new study suggests erythropoietin, now recognized as a potent neuroprotective cytokine, as a potential treatment for spinal cord injury.
\end{abstract}

S pinal cord injury (SCI) is one of the $\checkmark$ most frustrating maladies faced in neurology and medicine. The vast majority of SCI patients are young, and most survivors of significant injury face the prospects of limited recovery and permanent disability. The incidence of new SCI cases is high, exceeding 12,000 new cases per year of paraplegia or quadriplegia in the United States ${ }^{1}$. Yet with improved management, the mortality rate of SCI has steadily fallen. As a result, the prevalence of patients disabled by SCI now approximates 200,000 in the US alone. The need for effective acute intervention, both to limit the numbers of permanently impaired patients-and to give real hope to the newly injured-has never been greater.

Current treatment of SCI is limited to high-dose glucocorticoid therapy, which is useful only when adminis-

\section{STEVEN A. GOLDMAN ${ }^{1} \&$ MAIKEN NEDERGAARD ${ }^{2}$}

tered within hours of injury. The mechanisms by which the steroids exert their moderately beneficial effects remain unclear, though they are generally attributed to the anti-inflammatory effects of the glucocorticoids. Yet despite the fundamental inadequacy of highdose glucocorticoid treatment, it has remained the only available therapy for SCI.

In a recent issue of the Proceedings of the National Academy of Science USA, Gorio et al. report that the acute administration of erythropoietin (EPO) can attenuate the effects of acute SCI, and accelerate recovery after injury ${ }^{2}$. In two different experimental models of SCI in rats, one chosen to mimic brief crush injury, and the other a model of trau- matic contusion, EPO treatment markedly improved neurological recovery.

In the crush-injury model, in which the thoracic spinal cords of anesthetized rats were briefly compressed with a clip, untreated animals were initially paraplegic, and still barely able to move their hind limbs 4 weeks after injury. The authors quantified spinal cord injury using a standardized 21-point scoring system, called the BBB scale (on which 0 is plegic and 21 normal $)^{3}$. The untreated rats had mean BBB scores of 1 immediately after SCI, and of 10 when assessed 4 weeks later. In contrast, their treated counterparts, who received a single injection of EPO $(1,000 \mathrm{U} / \mathrm{kg})$ an hour after injury, improved to a BBB score of 18 at 4 weeks. Notably, a single injection of EPO at 1 hour was as effective as daily injections given for the first several days; accordingly, most of 